\title{
Madagascar, Accordéons et esprits ancestraux
}

Enregistrements, texte et photographies : Ron Emoff. Traduction française : Isabelle Schulte-Tenckhoff. 10 plages (59'14"). CD AIMP LXV, VDE-Gallo CD-1065, 2001.

\section{Julien Mallet}

\section{OpenEdition}

\section{Journals}

Édition électronique

URL : http://journals.openedition.org/ethnomusicologie/221

ISSN : 2235-7688

Éditeur

ADEM - Ateliers d'ethnomusicologie

Édition imprimée

Date de publication : 1 décembre 2001

Pagination : 348-349

ISBN : 2-8257-07-61-9

ISSN : 1662-372X

Référence électronique

Julien Mallet, "Madagascar, Accordéons et esprits ancestraux », Cahiers d'ethnomusicologie [En ligne],

14 | 2001, mis en ligne le 10 janvier 2012, consulté le 22 avril 2019. URL : http://

journals.openedition.org/ethnomusicologie/221

Ce document a été généré automatiquement le 22 avril 2019.

Tous droits réservés 


\title{
Madagascar, Accordéons et esprits ancestraux
}

\author{
Enregistrements, texte et photographies : Ron Emoff. Traduction \\ française : Isabelle Schulte-Tenckhoff. 10 plages (59'14"). CD AIMP LXV, \\ VDE-Gallo CD-1065, 2001.
}

Julien Mallet

\section{RÉFÉRENCE}

Madagascar, Accordéons et esprits ancestraux. Enregistrements, texte et photographies : Ron Emoff. Traduction française : Isabelle Schulte-Tenckhoff. 10 plages (59'14'). CD AIMP LXV, VDE-Gallo CD-1065, 2001.

1 Ce disque accueille des enregistrements réalisés par Ron Emoff entre 1993 et 1994 sur la côte Est de l'île de Madagascar, dans la région de Tamatave (Tamatave-ville et FénériveEst), d'Antananarivo (Ambohimanga) et à Diégo, avec des musiciens d'ethnies Betsimsaraka (5 plages), Antandroy (2), Merina (1), Antaisaka (1) et Sakalava (1).

2 Le livret est constitué d'une présentation générale d'un peu plus de trois pages. Les dix enregistrements sont ensuite commentés sur quatre pages et demie. Une carte de Madagascar et quatre photographies représentant les musiciens séparent la traduction française du texte original en anglais.

3 Dans la première partie de son introduction, l'auteur présente les caractéristiques de l'accordéon à boutons, le plus répandu à Madagascar, grâce à une comparaison didactique avec l'accordéon à touche. Échelle diatonique, technique de jeu, conception de la structure et du flux de la musique qu'il induit, accord et timbre sont autant d'éléments que Ron Emoff invoque pour en cerner la spécificité. La plupart des musiciens malgaches, nous dit-il, « affirment qu'il leur faut des types et des modèles particuliers d'accordéon, notamment en raison de la capacité de ces instruments de produire les tonalités particulières qui servent à communiquer avec les ancêtres ". Cette thèse forte, qui touche à des préoccupations cruciales en ethnomusicologie, est étayée par une comparaison avec 
les Cajuns du sud-ouest de la Louisiane - peut être au détriment d'une explication interne plus fouillée. L'auteur nous offre néanmoins, par la suite, plusieurs éclairages des rapports entre musique et esprits ancestraux à Madagascar, notamment à partir de descriptions empruntées à différentes pratiques rituelles. Lors des rites de possession Tromba,par exemple, « de nombreux esprits ne se manifesteront pas à moins qu'ils soient cajolés et apaisés à l'aide de leurs compositions musicales favorites». Une fois les ancêtres présents, la musique doit leur plaire et les retenir ${ }^{1}$.

La moitié des pièces de ce disque ont été exécutées pour les besoins de l'enregistrement. Seules trois plages témoignent de situations où accordéon et esprits ancestraux se côtoient réellement dans le cadre de rituels organisés à cet effet. A l'argument selon lequel la plupart des musiciens malgaches "s'impliquent dans la communication et l'interaction avec les esprits de leurs ancêtres chaque fois qu'ils font de la musique ", nous préférons le parti pris également revendiqué par l'auteur $\mathrm{d}$ '«illustrer le jeu de l'accordéon » en sélectionnant, entre autres, des enregistrements « hors contexte » et/ou privilégiant une prise de son focalisée sur l'instrument. De bons enregistrements nous donnent en effet accès à des moments musicaux forts où l'accordéon est bien mis en valeur comme dans la première (magnifique!) et la dernière plage attribuées aux musiciens Antandroy. Ce parti pris est peut-être plus discutable lorsque l'accordéon semble un peu lointain (plages 2 et 3) ou lorsque l'espace sonore est réellement amputé (plages 5, 6, 8 et 9) : chant, claquements de mains, interjections... sont à demi présents pour laisser place à un accordéon lui-même un peu lointain et désincarné.

5 Cependant, l'ensemble des enregistrements témoigne d'une vitalité, d'une inventivité et d'une richesse musicale réelles qui confirment l'intérêt profond des problématiques évoquées par Ron Emoff ${ }^{2}$ comme celles liées aux différents processus d'appropriation qu'il aborde d'un point de vue historique, en mettant l'accent sur l'originalité du jeu musical, ou encore à partir de l'« accordéon diatonique ", parfois volontairement modifié ${ }^{3}$ , aujourd'hui devenu « akordôgna », « gorodora » ou « hararavo » : corps joyeux...

\section{NOTES}

1. Pour une étude plus approfondie voir l'article de l'auteur du CD : «Clinton, Bush and Hussein in Madagascar ", the World of Music 42(2), 2000 : 51-73.

2. Les enregistrements ont été effectués dans le cadre d'une recherche de deux ans à Madagascar destinée notamment à examiner les interrelations entre la pratique musicale, les cultes de possession, la mémoire du passé et le colonialisme. Ce travail est l'objet d'une publication (sous presse): Recollecting from the Past: Musical Practice and Spirit Possession on the East Coast of Madagascar. Wesleyan University Press, Music and Culture Series.

3. Comme le note Victor Randrianary dans son disque «Madagascar, pays Masikoro, l'accordéon" (Ocora) et comme j'ai pu le constater lors de mes recherches dans la région de Tuléar. Dans cette région de l'île (Sud-ouest), l'accordéon nommé hararavo est dit " castré » ( famosira) par un procédé qui consiste à en limer les lames. 